\title{
Spin polarization in semimagnetic semiconductor two-barrier spin filters
}

\author{
S.B. Lev, V.I. Sugakov, G.V. Vertsimakha \\ Institute for Nuclear Research, NAS of Ukraine \\ 47, prospect Nauky, 03680 Kyiv, Ukraine
}

\begin{abstract}
The spin-dependent tunneling of electrons through the CdMgTe-based twobarrier resonant tunneling system with a semimagnetic CdMnTe well is studied. The level splitting in the semimagnetic well under an external magnetic field, caused by giant Zeeman splitting, allows one to achieve a high level of spin polarization of the current flowing through the spin filter. The current polarization degree depending on different parameters of the system such as the carrier density, concentration of magnetic ions, temperature, and the strength of the external magnetic and electric fields is analyzed.
\end{abstract}

Keywords: resonant tunneling diode, spin filter, semimagnetic semiconductor.

Manuscript received 08.09.07; accepted for publication 19.12.07; published online 31.01.08.

\section{Introduction}

During last few years, different facilities and methods of creation of systems with a considerable level of electron spin polarization were intensively studied because of the possible applications [1-3]. Thus, it is important to create appropriate spin filters for the polarized spin injection into an investigated system. There are two most popular systems for the spin polarization in semiconducting crystals. One of the candidates is the asymmetric nonmagnetic heterostructure based on the interface-induced Rashba spin-orbit coupling [4, 5]. Another possibility to create the spin filter is based on the effect of the spin dependent electron tunneling through semimagnetic semiconductor double-well structures. Such CdTe-based two-barrier system with semimagnetic barriers and well was proposed and theoretically investigated in work [6]. In semimagnetic semiconductors, the strong exchange interaction between the carriers and the localized spins of magnetic ions (giant Zeeman splitting) leads to a strong dependence of the energy of the resonant tunneling level on the external magnetic field and the spin of carriers. For a certain range of parameters of the system in an applied external electric field, two clear peaks of the current are observed experimentally [7]. Recently, similar systems with different configurations of barriers, for example twoand one-barrier spin filters, become very popular [8-16].

As distinct from work [6], we consider the CdTebased resonant tunneling structure, in which magnetic impurities $\mathrm{Mn}$ are placed in the well and the barriers formed by the layers containing nonmagnetic impurities, Mg ions. System, in which Mg impurities are localized in barriers, allows one to tune the relative position of the resonant level in the well and the Fermi level outside the barriers, which leads to a higher degree of spin polarization. In the present work, the polarization degree is investigated as a function of the system parameters: the charge carrier concentration, the size of the spin filter well and barriers, temperature, and so on. Such an analysis allows performing the system parameters optimization and obtaining the highest current and electron spin polarization.

\section{The system under consideration}

In the present work, we study the two-barrier semimagnetic semiconductor resonant tunneling structure $\mathrm{Cd}_{1-\mathrm{y}} \mathrm{Mg}_{\mathrm{y}} \mathrm{Te} / \mathrm{Cd}_{1-\mathrm{z}} \mathrm{Mg}_{\mathrm{z}} \mathrm{Te} / \mathrm{Cd}_{1-\mathrm{x}} \mathrm{Mn}_{\mathrm{x}} \mathrm{Te} / \mathrm{Cd}_{1-\mathrm{z}} \mathrm{Mg}_{\mathrm{z}} \mathrm{Te} /$ $\mathrm{Cd}_{1-\mathrm{y}} \mathrm{Mg}_{\mathrm{y}} \mathrm{Te}$, where $y<z$. The general design of such a system and its band structure are shown in Fig. 1. Magnetic properties of the system are determined by the semimagnetic semiconductor CdMnTe layer that forms a quantum well in the structure. The concentration of charge carriers in the external layers of the system under study is created by the doping of the pre-barrier region. The Fermi energy is marked in the figure as $E_{\mathrm{F}}$. The electric and external magnetic fields are applied along the crystal growth direction $z$. A giant Zeeman splitting leads to a splitting of the resonant level in the 


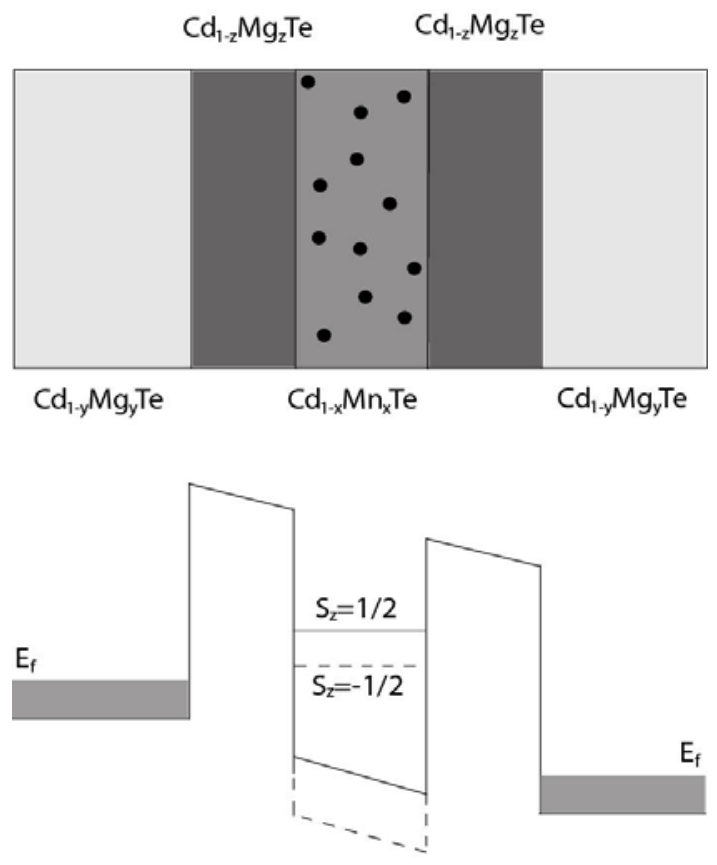

Fig. 1. Schematic diagram of the heterostructure and the band alignment for spin-up and the spin-down electrons in a magnetic field. Thin lines indicate the resonance levels for spin-up (solid) and the spin-down (dashed) electrons.

semimagnetic layer, and thus the transition coefficient depends on the electron spin. We consider that the voltage drops mainly in the device region. First of all, we will calculate the current polarization relying on the transmission coefficients of electrons with different spin orientations.

The Schrödinger equation for an electron in such a system has the form

$$
\begin{aligned}
& {\left[\frac{1}{2 m_{e}}\left(\left(\hat{p}_{x}+e B y\right)^{2}+\hat{p}_{y}^{2}+\hat{p}_{z}^{2}\right)+V(z, B, E)\right] \times} \\
& \times \tilde{\Psi}(x, y, z)=\tilde{E} \tilde{\Psi}(x, y, z),
\end{aligned}
$$

where $m_{e}$ is the electron mass, $B$ is the magnetic field, $V(z, B, E)=V_{\mathrm{x}}(\mathrm{z})+V_{\mathrm{B}}(\mathrm{z})+V_{E}(\mathrm{z})$ is the potential which describes the crystal lattice deformation caused by the replacement of cations by the magnetic impurity ions $V_{\mathrm{x}}(\mathrm{z})$, the exchange interaction $V_{\mathrm{B}}(\mathrm{z})$, and the influence of the applied external electric field $V_{E}(z)$, correspondingly. The electron current outside the spin filter is calculated on the base of the electron transition coefficient of the system of semiconductor barriers. Taking into account the fact that the potential $V(z, B, E)$ depends on the variable $z$ only and using the standard methods of calculations (e.g., see work [6]), we obtain the spin-dependent electron transition coefficient in the applied external magnetic and electrical fields as

$$
D_{\sigma}\left(U, H, E_{\perp}\right)=\frac{k_{5}}{k_{1}}|T|^{2} .
$$

The carrier current density is given by the formula

$$
\begin{aligned}
& j_{\sigma}(U, H)=\frac{e^{2} B}{4 \pi \hbar^{2}} \sum_{n} \int d E_{\perp} D_{\sigma}\left(U, H, E_{\perp}\right) \times \\
& \times\left\{f\left[E_{\perp}+\left(n+\frac{1}{2}\right) \hbar \omega_{H}\right]-f\left[E_{\perp}+\left(n+\frac{1}{2}\right) \hbar \omega_{H}+e U\right]\right\},
\end{aligned}
$$

where $f\left(E_{\perp}\right)$ is the Fermi-Dirac distribution function. By definition, the current polarization is determined as

$$
P_{j}=\frac{j_{\sigma^{+}}(U, H)-j_{\sigma^{-}}(U, H)}{j_{\sigma^{+}}(U, H)+j_{\sigma^{-}}(U, H)} .
$$

\section{The current polarization}

The spin-dependent electron transition coefficient in the two-barrier resonant tunneling structure $\mathrm{Cd}_{1-y} \mathrm{Mg}_{\mathrm{y}} \mathrm{Te} /$ $\mathrm{Cd}_{1-\mathrm{z}} \mathrm{Mg}_{\mathrm{z}} \mathrm{Te} / \mathrm{Cd}_{1-\mathrm{x}} \mathrm{Mn}_{\mathrm{x}} \mathrm{Te} / \mathrm{Cd}_{1-\mathrm{z}} \mathrm{Mg}_{\mathrm{z}} \mathrm{Te} / \mathrm{Cd}_{1-\mathrm{y}} \mathrm{Mg}_{\mathrm{y}} \mathrm{Te}$, was calculated in the case where the electron effective mass $m_{e}=0.096 m_{0}$, the dielectric constant $\varepsilon=9.7$, the exchange integral $N_{0} \alpha=0.22 \mathrm{eV}$ and $Q_{V}=0.6$ [17]. The shape and the magnitude of the general potential is determined as a sum of the components $V_{\mathrm{x}}(\mathrm{z}), V_{B}(\mathrm{z})$, and $V_{E}(z)$, where $V_{H}(z)$ was calculated according to the empirical formula presented in [18], and $V_{x}(z)$ was calculated by the equations $E_{g}\left(\mathrm{Cd}_{1-\mathrm{x}} \mathrm{Mg}_{\mathrm{x}} \mathrm{Te}\right)=$ $[1.606(1-x)+3.6 x-0.3 x(1-x)] \mathrm{eV}$ and $E_{g}\left(\mathrm{Cd}_{1-\mathrm{x}} \mathrm{Mg}_{\mathrm{x}} \mathrm{Te}\right)=$ $[1.606(1-x)+3.198 x] \mathrm{eV}$ with relative concentrations of the impurities $\mathrm{Mn}$ and $\mathrm{Mg}$ characterized by $x=0.05, y=$ $0.06, z=0.12$. We chose the barriers widths $L_{1}=L_{2}=$ $70 \AA$ and the well width, respectively, $d=60 \AA$.

Figure 2 shows the total current of electrons against the applied voltage for the different values of the external magnetic field $B=0.5,2$, and $4 \mathrm{~T}$, the donor concentration $N_{D}=5 \cdot 10^{22} \mathrm{~m}^{-3}$, and the temperature $T=$ $2 \mathrm{~K}$. Each peak of the total current corresponds to the peak of the current with a certain electron spin polarization. The results of calculations of different components of the tunneling current for the donor concentration in the external layers of the structure equal to $N_{D}=5 \cdot 10^{22} \mathrm{~m}^{-3}$, which corresponds to the Fermi level $E_{f} \approx 5 \mathrm{meV}$, for the magnetic field $B=0.5 \mathrm{~T}$ and the temperature $T=2 \mathrm{~K}$ are shown in Fig. 2 by dotted curves. These curves show that, by changing the applied electric field, we can reach the situation where the current of electrons with a certain spin polarization will be greater by a few orders of magnitude than the current with the opposite spin polarization. According to the numerical calculation of the current polarization, we have a zero polarization for the magnetic field $B=0 \mathrm{~T}$. Starting from $B=0.5 \mathrm{~T}$, there exist some values of the bias, at which the value of polarization can reach $90 \%$. The range of the electric field, at which current polarization exceeds any demanded level (e.g., $90 \%$ ), will growth when the external magnetic field increases. 


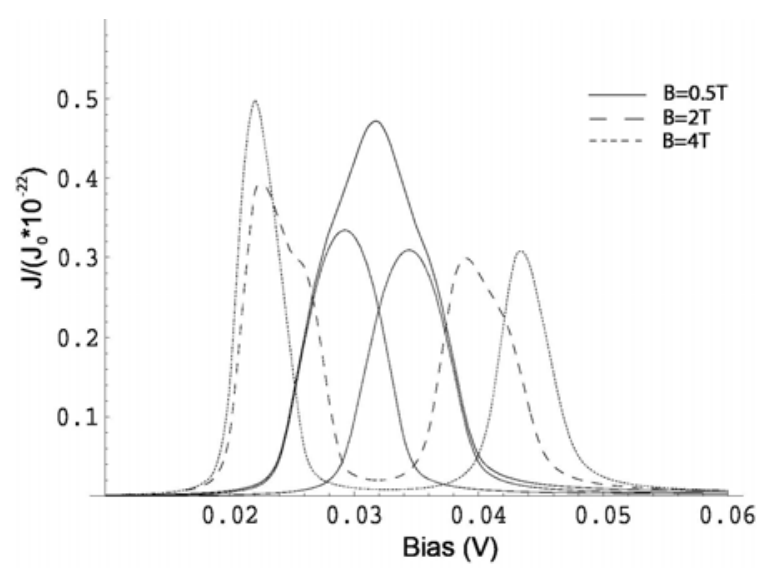

Fig. 2. Current densities for spin-up and spin-down electrons and the total current for the different values of the external magnetic field as a function of the applied bias, $J_{0}=e^{2} / 4 \pi^{2} \hbar^{2}$. Dotted curves correspond to the current components with different spin polarizations of carriers for $B=0.5 T$.

Figure 3 shows the total current under the applied voltage for the different temperatures $T=5,10$, and $15 \mathrm{~K}$, the external magnetic field $B=4 \mathrm{~T}$, and the donor concentration in the leftmost layer $N_{D}=5 \cdot 10^{22} \mathrm{~m}^{-3}$. The figure shows that the peaks become wider when the temperature increases, and the correspondent maxima can overlap at a certain temperature. This behavior of the current of electrons with different spin orientations is explained by the distribution function dependence on the temperature because the greater temperature enlarges the range of the applied external electric field, in which there are the electrons in the conduction band of the leftmost layer with the energy corresponding to the resonant tunneling energy. In other words, the electron distribution change with increase in the temperature will override the phenomena caused by the splitting of resonant tunneling levels in the external magnetic field. For example, at some values of the structure parameters and external fields, the maximum value of the current polarization at the temperature $T=5 \mathrm{~K}$ can be $P_{j}>0.99$. But, at the temperature $T=20 \mathrm{~K}$, it does not exceed 0.5. A similar behavior of the current polarization as a function of temperature was observed in work [7] for a ZnSe-based system. The smaller experimental value of the separation of peaks can be explained by the greater concentration of carriers, which also agrees with our results presented in Fig. 3.

All above-presented figures describe the current dependence on the parameters which change the location of the peaks of the currents of electrons with different spin orientations but do not change the value of the current essentially. Fig. 4 shows the dependence of the total current of electrons on the applied voltage for several different values of the donor concentration $N_{D}=$ $5 \cdot 10^{22}, 1 \cdot 10^{23}$, and $1.5 \cdot 10^{23} \mathrm{~m}^{-3}$, at the temperature $T=$ $2 \mathrm{~K}$, and the external magnetic field $B=6 \mathrm{~T}$. These concentrations correspond to the different energies of the Fermi level: $E_{\mathrm{F}} \sim 5,10$, and $15 \mathrm{meV}$, respectively. It is

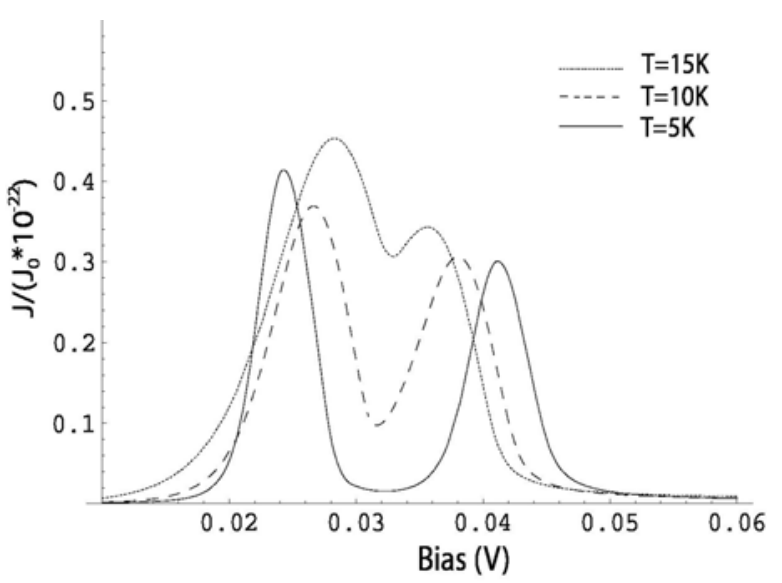

Fig. 3. Total current for different temperatures as a function of applied voltage, $J_{0}=e^{2} / 4 \pi^{2} \hbar^{2}$.

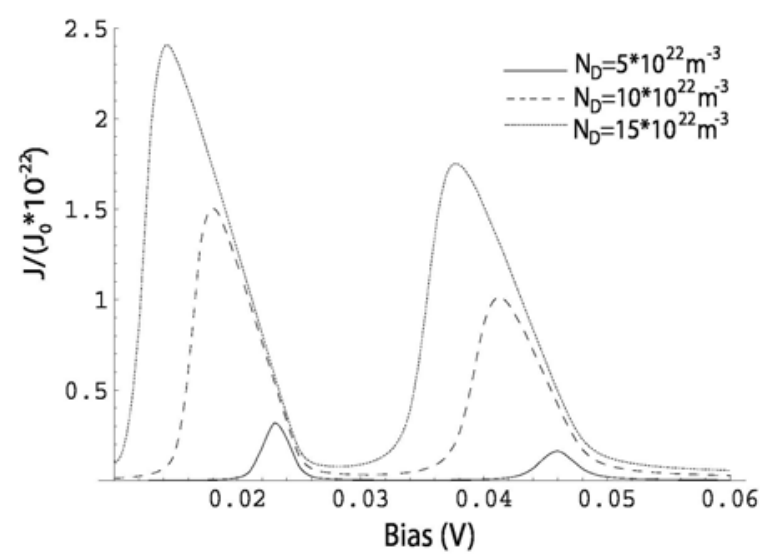

Fig. 4. Total current for the different values of the donor concentration (the Fermi level energy) as a function of the applied bias, $J_{0}=e^{2} / 4 \pi^{2} \hbar^{2}$.

clearly seen from the figure that the absolute value of the current increases when the charge carrier concentration grows. Fig. 4 also indicates that increasing the donor concentration makes peaks wider and closer one to another. This behavior can be explained similarly as above, because the greater energy of the Fermi level enlarges the interval of applied external electric fields, at which there are electrons with corresponding energy in the pre-barrier region of the system. As shown above, the current polarization is very sensitive to the donor concentration. The current polarization also depends on a number of parameters of the system such as the widths of the barriers and the well, the concentration of nonmagnetic $\mathrm{Mg}$ ions in the barriers and magnetic ions in the well layer (the greater concentration of magnetic Mn ions in the well corresponds to the larger splitting of resonant tunneling levels and, consequently, to the better localization of peaks and current polarization). For example, increasing the barrier width from $L_{1}=L_{2}=$ $50 \AA$ to $L_{1}=L_{2}=90 \AA$ does not change the location of the corresponding peaks of the current, but decreases its maximum value more than by a factor of five.

\section{(C) 2007, V. Lashkaryov Institute of Semiconductor Physics, National Academy of Sciences of Ukraine}


Parameters of the system are chosen to obtain the maximum polarization while minimizing the barrier deformation caused by the external electric field.

\section{The charge carrier polarization}

The spin filter described above allows the passage of a highly polarized current of electrons. But the interaction of electrons with the surrounding material leads to the electron relaxation and to a decrease of the spin polarization. The distance, at which the spin polarization remains significant, is determined by the properties of a crystal, such as the spin relaxation time, the electron mobility, and so on. The degree of polarization is the relative difference of concentrations of electrons with different spin projections,

$P=\frac{n_{+}-n_{-}}{n_{+}+n_{-}}$

where $n_{+}$and $n_{-}$are the concentrations of electrons with $s_{z}=\frac{1}{2}$ and $s_{z}=-\frac{1}{2}$, respectively. Spin-polarized electrons injected into a semiconductor lose the spin polarization degree due to various spin relaxation processes. Here, we suppose that the equilibrium state with respect to energy is established more faster than that with respect to spin. Thus, the density of electrons with different spin orientations and their polarization can be calculated in the two-component drift-diffusion transport model [19] and can be described by the continuity equations

$\frac{\partial n_{ \pm}}{\partial t}=\frac{1}{e} \operatorname{div} j_{ \pm}+\frac{n_{\mp}}{\tau_{\mp}}-\frac{n_{ \pm}}{\tau_{ \pm}}$,

where $\tau_{ \pm}$and $\tau_{\mp}$ are the relaxation times for electrons with the spin $s_{z}=\frac{1}{2}$ and $s_{z}=-\frac{1}{2}$, respectively, the time of the electron transition from the state with spin $s_{z}=-\frac{1}{2}$ into the one with $s_{z}=-\frac{1}{2}$ and vice versa. We note that the current density of electrons with different spin polarizations $j_{ \pm}=e \mu n_{ \pm} E+e D \frac{d n_{ \pm}}{d z}$, where $D$ is the diffusion coefficient, $\mu$ is the electron mobility and $D=\mu k_{B} T / e$. In the steady state, we have the system of equations

$$
\left\{\begin{array}{l}
D \frac{d^{2} n}{d z^{2}}+\mu E \frac{d n}{d z}+n \mu \operatorname{div} E=0 \\
D \frac{d^{2} p}{d z^{2}}+\mu E \frac{d p}{d z}+p \mu \operatorname{div} E-p\left(\frac{1}{\tau_{-}}+\frac{1}{\tau_{+}}\right) \\
+n\left(\frac{1}{\tau_{-}}-\frac{1}{\tau_{+}}\right)=0
\end{array}\right.
$$

where $n=n_{+}+n_{-}$and $p=n_{+}-n_{-}$. According to the equation for the electron current density, the boundary conditions for the total concentration and for the difference of electron concentrations take the form

$$
\begin{aligned}
& j_{ \pm}(0) \pm j_{\mp}(0)= \\
& =e \mu\left(n_{+}(0) \pm n_{-}(0)\right) E(0)+\left.e D \frac{d\left(n_{+} \pm n_{-}\right)}{d z}\right|_{z=0},
\end{aligned}
$$

where the origin of coordinates $z=0$ is located at the external boundary of the second barrier, and the values $j_{+}(0)$ and $j_{-}(0)$ calculated above are the currents densities of electrons with different spin orientations after passing the second barrier. We suppose as well that, for a low temperature, the spin distribution at infinity is normal, $p(\infty) \approx 0$, and the total carrier concentration is equal to the donor concentration $n(\infty)=N_{D}$.

In order to obtain a full system of kinetic equations for the electron density in the conduction band, Eq. (7) must be complemented by the Poisson equation $\frac{d E}{d z}=\frac{e}{\varepsilon \varepsilon_{0}}(N-n)$. Both the Poisson equation and the equation for the total current give the equation for the electric field strength:

$$
\frac{d^{2} E}{d z^{2}}+\frac{e}{k_{B} T} E \frac{d E}{d z}-\frac{e^{2} N}{\varepsilon \varepsilon_{0} k_{B} T} E+\frac{j}{\varepsilon \varepsilon_{0} D}=0,
$$

where $j=j_{+}+j_{-}(0)$ is the total current. The boundary conditions for the electric field strength are formulated according to the following physical considerations. We suppose that the electric field at the point $z=0$ is continuous and is equal to the electric field inside the barriers which is determined by the voltage drop $U$ on the inner layers of the structure $E(0)=U /\left(L_{1}+d+L_{2}\right)$. On the other hand, the electric field at infinity is constant, so Eq. (9) gives $E(\infty)=j /\left(\mu\right.$ e $\left.N_{D}\right)$. Hence, the system of equations (7) and (9) with boundary conditions completely determines the spin polarization of the electrons in the layer placed after the last barrier.

Figure 5 shows the dependences of spin and current polarizations on the distance from the boundary of the barrier for several values of the relaxation time and for the electron mobility $\mu=10^{-2} \mathrm{~m}^{2} \mathrm{~s} / \mathrm{V}$. The spin-lattice relaxation time depends on the sample conditions (the presence of impurities, the electron-phonon interaction, etc.) and can reach the nanosecond range in II-VI semiconductors $[3,20]$. It is clearly seen that, for the given parameters, the electron spin polarization can exceed the value of $90 \%$ and would decrease when the distance from the spin filter grows. The width of the layer with large spin polarization is determined, first of all, by the value of the electron spin relaxation time for the given system. 


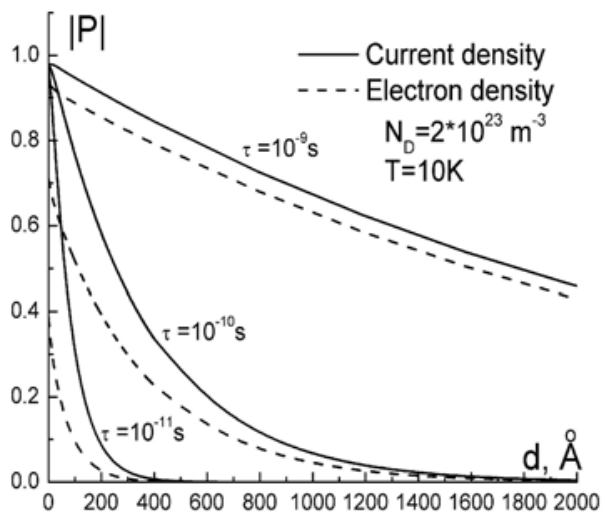

Fig. 5. Spin density and current density polarization degrees as a function of the distance from the boundary of a barrier.

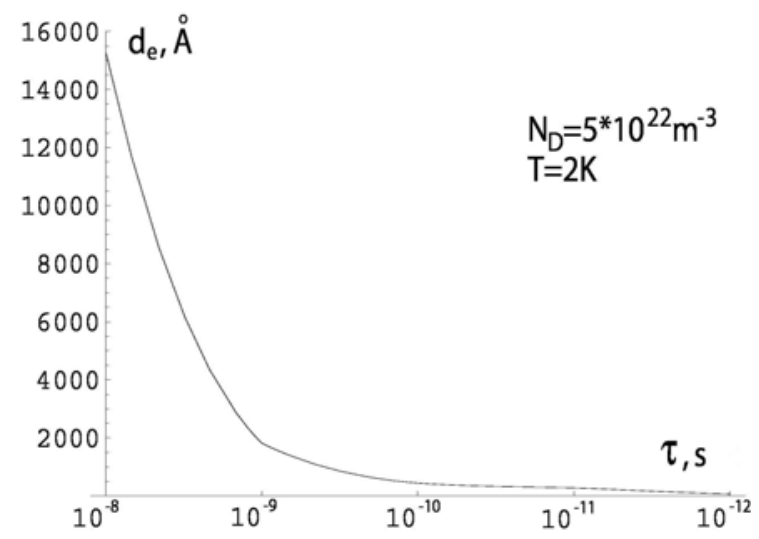

Fig. 6. Distance from the boundary of the barrier, at which the polarization of carriers decreases by $e$ times as a function of the electron spin relaxation time at $U=0.022 \mathrm{~V}$.

Figure 6 demonstrates how the distance from the barrier, at which a high degree of polarization of carriers exists, depends on the electron spin relaxation time. In Fig. 6, $d_{e}$ stands for the distance from the barriers, at which the polarization of carriers decreases by a factor of $e, P(0) / P\left(d_{e}\right)=e$. Figures 5 and 6 indicate that the penetration depth of the polarization critically increases with the electron spin relaxation time. For the relaxation time exceeding $10^{-9}$, the polarized region can spread for several microns. The calculations also showed that the penetration depth increases when the electron mobility grows.

We also calculated the spin and current polarizations in a simpler one-barrier semimagnetic semiconductor spin filter. The magnetic properties of such a system are determined by the $\mathrm{Cd}_{1-\mathrm{x}} \mathrm{Mn}_{\mathrm{x}} \mathrm{Te}$ layer that forms the barrier. For electrons with the spin projection $s_{z}=\frac{1}{2}$ and $s_{z}=-\frac{1}{2}$, the heights of the barrier in the magnetic field are different. The splitting of the barrier height leads to the splitting of the transition coefficient for electrons with different spin projections. Therefore, different values of the current for the different electron spin polarizations are obtained. In the case of a one-barrier spin filter, the current and carrier polarizations can reach considerable values close to $50 \%$ that is less than that in the case of resonant tunneling.

\section{Conclusion}

We have studied the two-barrier resonant tunneling CdTe-based system with $\mathrm{Mg}$ ions in barriers and $\mathrm{Mn}$ ions in the well placed in the external electric and magnetic fields. The dependences of the polarization degree on the temperature, concentration of magnetic ions, the well width, and the doping level are determined. It is shown that, for certain values of the system parameters and the external field strengths, the polarization higher than $90 \%$ can be obtained. The degree of carrier polarization was studied as a function of the distance from the barrier. It is shown that the distance, at which a high degree of the polarization of carriers (above $50 \%$ ) is maintained, can reach several microns.

\section{References}

1. D.P. DiVincenzo // Science 270, p. 255 (1995).

2. B.E. Kane // Nature 393, p. 133 (1998).

3. J.M. Kikkawa, I.P. Smorchkova, N. Samarth, D.D. Awschalom // Science 277, p. 1284 (1997).

4. Y.-X. Li, Y. Guo, B.-Z. Li // Phys. Rev. B 71, 012406 (2005).

5. O. Bleibaum // Phys. Rev. B 71, 195329 (2005).

6. V.I. Sugakov, S.A. Yatskevich // Sov. Tech. Phys. Lett. 18, p. 134 (1992)

7. A. Slobodskyy, C. Gould, T. Slobodskyy et al. // Phys. Rev. Lett. 90, 246601 (2003)

8. S. Nonoyama, J. Inoue // Physica E 10, p. 283 (2001).

9. X. Zhang, B.Z. Li, G. Sun, F.C. Pu // Phys. Lett. A 245, p. 133 (1998).

10. J.C. Egues // Phys. Rev. Lett. 80, p. 4578 (1998).

11. J.C. Egues, C. Gould, G. Richter, L.W. Molenkamp // Phys. Rev. B 64, 195319 (2001).

12. Y. Guo, B.-L. Gu, H. Wang, Y. Kawazoe // Phys. Rev. B 63, 214415 (2001).

13. K. Chang, J.B. Xia, F.M. Peeters // Phys. Rev. B 65, 115209 (2002).

14. N.N. Beletskii, G.P. Berman, S.A. Borysenko // Phys. Rev. B 71, 125325 (2005).

15. P. Havu, N. Tuomisto, R. Vaananen, M.J. Puska, R.M. Nieminen // Phys. Rev. B 71, 235301 (2005).

16. Y. Guo, J.-Q. Lu, B.-L. Gu et al. // Phys. Rev. B 64, 155312 (2001).

17. W. Ossau, R. Fiederling, B. Konig et al. // Phys. Low.-Dim. Struct. 11/12, p. 89 (1997).

18. J.A. Gaj, W. Grieshaber, C. Bodin-Deshayes et al., // Phys. Rev. B 50, p. 5512 (1994).

19. S. Saikin, Yu.V. Pershin, V. Privman // condmat/0504001.

20. M. Bejar, D. Sanches, G. Platero et al. // Phys. Rev. B 67, 045324 (2003). 